\title{
Tecnologias para a Produção de Biofertilizantes: tendências e oportunidades
}

\author{
Technologies for the Production of Biofertilizers: trends and \\ opportunities
}

\author{
Eduardo Cardoso Garrido ${ }^{1}$ \\ Ângela Machado Rocha ${ }^{2}$ \\ Douglas Alves Santos ${ }^{3}$ \\ Jose Miguel Vicente Gomila \\ ${ }^{1}$ SEBRAE/BA; UFBA; SENAI CIMATEC, Salvador, BA, Brasil. \\ ${ }^{2}$ Universidade Federal da Bahia, Salvador, BA, Brasil. \\ ${ }^{3}$ Instituto Nacional da Propriedade Industrial - INPI, Salvador, BA, Brasil. \\ ${ }^{4}$ Universitat Politècnica de València, Espanha
}

\begin{abstract}
Resumo
Biofertilizante é aquele fertilizante que dispõe de microrganismos vivos, dentre eles bactérias e fungos, que contribuem para a fertilidade do solo. $\mathrm{O}$ artigo objetiva analisar os dados históricos de patentes de modo a determinar quais as principais instituições mundiais que investem em pesquisa e desenvolvimento tecnológico relacionados aos biofertilizantes. A Metodologia desenvolve o tratamento e análise dos resultados de patentes relacionadas aos biofertilizantes por meio do Vantage Point, software de mineração de textos que se utiliza de um dicionário de palavras afins para agrupar autores, instituições e termos. Japão, Europa (EPO), Estados Unidos, Coreia, Índia e Brasil encontram-se entre os escritórios de patente com proeminência no âmbito de publicações de patentes. Dentre os dados levantados, destacam-se nos resultados as seguintes instituições japonesas: Hitachi Ltd; Sanyo Electric Co Ltd; Mitsubishi Electric Corp. Do total de titulares de patentes, 95,53\% respondem por Empresas Privadas e 3,17\% por Institutos de Pesquisa.
\end{abstract}

Palavras-chave: Patentes. Fertilizantes. Vantage Point.

\begin{abstract}
Biofertilizer is a fertilizer that has living microorganisms, among them bacteria and fungi, that contribute to soil fertility. The objective of this paper is to analyze historical patent data to determine the main world institutions that invest in research and technological development related to biofertilizers. The Methodology develops the treatment and analysis of the results of patents related to biofertilizers through Vantage Point, text mining software that uses a dictionary of related words to group authors, institutions and terms. Japan, Europe (EPO), the United States, Korea, India and Brazil are among the patent offices with prominence in the field of patent publications. Among the data collected, the following Japanese institutions stand out in the results: Hitachi Ltd; Sanyo Electric Co Ltd; Mitsubishi Electric Corp. Of the total number of patent holders, $95.53 \%$ account for Private Companies and 3.17\% for Research Institutes.
\end{abstract}

Keywords: Patents. Fertilizers. Vantage Point.

Área tecnológica: Prospecções Tecnológicas de Assuntos Específicos. 


\section{Introdução}

O termo cunhado biofertilizante é proveniente dos termos fertilizante biológico. Refere-se ao fertilizante que dispõe, em sua composição, de microrganismos vivos, dentre elas bactérias e fungos, que contribuem para a fertilidade do solo por meio da fixação do nitrogênio atmosférico, da solubilização do fósforo, do potássio, do zinco e de outros macro e micro nutrientes para um melhor desenvolvimento dos fito-hormônios e absorção de nutrientes necessários ao crescimento das plantas (BARMAN e outros, 2017; IAEA, 2018).

Os biofertilizantes apresentam contribuições para o meio ambiente, fruto da promoção de um menor consumo de fertilizantes tradicionais e pesticidas. O seu processo produtivo, natural, apresenta consigo uma redução do consumo de combustíveis fósseis e de compostos químicos utilizados no processo produtivo dos fertilizantes convencionais.

Por meio da reutilização dos resíduos como matéria-prima para a composição dos biofertilizantes, tem-se o decréscimo da quantidade de matéria orgânica disposta em lixões e aterros, com benefícios para a agricultura e para o fechamento do ciclo de nutrientes. São exemplos de fontes de insumo para os biofertilizantes: os resíduos de colheita, resíduos de matadouro, resíduos sólidos municipais, excretas de humanos, gado, porcos, aves, bodes e ovelhas.

Há também uma minimização das emissões de gases do efeito estufa, da contaminação de corpos d'água e dos impactos ambientais. Gera, consigo, benefícios econômicos quanto a oportunidades de renda e disponibilidade de fontes de energia com um menor custo, já que disponibiliza para as plantas os nutrientes já presentes no solo e na atmosfera (NGUMAH e outros, 2013).

O relatório Global Biofertilizer Market 2016-2020 estima uma taxa de crescimento anual para o mercado global de biofertilizantes de $13,9 \%$ até o ano de 2020 , com previsão de alcance de US\$ 1,66 bilhões até 2022 e uma produção acima de 1.200 kt em 2024. Tais valores se devem especialmente à demanda pelo mercado por produtos com certificação orgânica e pela exigência de uma maior produtividade por área de cultivo (BUSINESS WIRE, 2018; GLOBAL MARKET INSIGHTS, 2018).

O custo mais acessível dos fertilizantes biológicos apresenta-se como uma grande vantagem quando comparado ao fertilizante tradicional. As dificuldades, por outro lado, encontram-se nos altos custos iniciais de investimento, na baixa conscientização sobre os benefícios do uso de fertilizantes biológicos pelos agricultores, no tempo de validade restrito dos biofertilizantes, nas necessidades de treinamento de aplicadores e de pesquisas relacionadas às especificidades de cada tipo de cultivo (BUSINESS WIRE, 2018; BARMAN e outros, 2017) .

O setor de fertilizantes é estratégico para o país, já que o Brasil não tem produzido o suficiente para a sua demanda interna, com a necessidade de importação de fertilizantes, dependente assim de fatores e insumos externos. Esta demanda é responsável por cerca de um terço do déficit da indústria química. A indústria de fertilizantes divide-se basicamente em 3 (três) atividades distintas: produção de matéria-prima básica e matérias intermediárias, e de fertilizantes básicos e misturas. Aspectos como a disponibilidade energética e de insumos, infraestrutura portuária e de armazenamento, questões tecnológicas, regulatórias, tributárias e ambientais devem ser alvo de investimentos (DA COSTA; E SILVA, 2012; NETTO; DIAS, 1984). 
Neste mesmo cenário, a patente, como um ativo de propriedade intelectual, pode servir como um dos norteadores para investimentos em processos, produtos, serviços, marketing $e$ aspectos organizacionais, pilares da inovação. A prospecção tecnológica é um aspecto da aplicação de inteligência competitiva, já que o respectivo tratamento de dados patentários permite orientar as partes interessadas quanto aos grandes players do mercado, a direção adotada pelas empresas, em que países estão se preocupando em depositar patentes, dentre outras atitudes, guiando assim negócios e políticas públicas.

Uma patente é um direito exclusivo concedido pelo Estado relativamente a uma invenção (ou modelo de utilidade), que atende ao requisito de novidade, envolve uma atividade inventiva (ou ato inventivo) e é suscetível de aplicação industrial (INPI, 2013).

Pelo fato de as patentes disporem de informações técnicas quanto a invenções desenvolvidas (descrição dos inventos, definição de quais as instituições que estão desenvolvendo tecnologias e países em que são solicitadas reivindicações de prioridade), o monitoramento por patentes torna-se, desse modo, uma ferramenta informacional de tomada de decisão que promove uma melhor inserção de tecnologias na cadeia produtiva.

Os dados resultantes do monitoramento permitem, assim, apontar tendências e cenários para investimentos em tecnologia e planejamento no âmbito dos biofertilizantes.

O Vantage Point (VP) é um software de mineração de textos - text mining - para descoberta de tendências em praticamente qualquer banco de dados de texto estruturado. A ferramenta utiliza um dicionário de palavras afins (tesauros) para agrupar autores, instituições e termos mediante o NLP - Natural Language Processing. Esta ferramenta demonstra uma maior potencialidade de compatibilização e cruzamento de parâmetros como ferramenta de inteligência competitiva voltada para a análise de oportunidades de negócio.

Em razão do grande potencial do setor de agronegócio brasileiro, o presente trabalho desenvolveu, por meio do software VP, uma análise de famílias de patentes associadas à produção de biofertilizantes.

O objetivo do artigo é analisar os dados históricos de publicação de patentes do Japão, Europa, Estados Unidos, Brasil e Coreia de modo a determinar quais as principais instituições mundiais nesta temática sob o ponto de vista de investimentos em pesquisa e desenvolvimento (P\&D) tecnológico relacionados aos biofertilizantes.

\section{Metodologia}

A abordagem metodológica e as etapas de investigação são promovidas conforme as seguintes etapas:

a) Pesquisa inicial por meio do levantamento bibliográfico de publicações, artigos, capítulos de livros correlatos à temática por meio: da ferramenta de Pesquisa Qualis Periódicos, da Plataforma Sucupira, do Portal de Periódico CAPES; da SciELO; do Google Scholar; da Science Direct; de sites Institucionais e de Eventos;

b)Definição de palavra-chave para pesquisa: biofertilizer;

c) Acesso à base de dados de patentes relacionadas à biofertilizantes por meio do VP. Primeiro 
realizou-se uma pesquisa com o termo biofertilizer, referente à busca para biofertilizantes;

d) Obtenção dos resultados de publicações de patentes;

e) Tratamento dos dados por instituições, autores e termos;

f) Cruzamento dos referidos dados com os anos. Este é o ponto forte do Patent Vantage Point, o de permitir realizar análises exatas, pois primeiro se realiza a limpeza dos dados antes de se proceder o seu cruzamento;

g) Análise preliminar dos resultados obtidos;

h) Proposição de oportunidades para futuros trabalhos.

\section{Resultados e Discussão}

O trabalho apresenta os resultados do acesso à base de dados de patentes relacionadas à produção de biofertilizantes por meio do VP.

No âmbito da reutilização de resíduos para a produção de fertilizantes, grande parte dos resultados apresentaram-se relacionadas à fabricação de produtos químicos básicos, fertilizantes e compostos de nitrogênio, plásticos e borracha sintética em formas primárias com o código de classificação International Patent Classification (IPC) C05F, seguido pelo código de classificação IPC B09B. A IPC é o sistema de classificação internacional estruturado em classes, subclasses, grupos principais e subgrupos, criada a partir do Acordo de Estrasburgo (1971), totalizando 70 mil grupos (INPI, 2018).

Conforme relação constante no Inventário WIPO (2017), a codificação IPC C05F corresponde a biofertilizantes não cobertos pelas subclasses $\mathrm{C} 05 \mathrm{~B}$ e $\mathrm{C} 05 \mathrm{C}$, a exemplo de fertilizantes provenientes de resíduos ou rejeitos. A codificação IPC B09B corresponde à disposição de resíduos sólidos.

A Figura 1 relaciona os principais perfis com publicações de patentes neste âmbito: Japão, Europa, Estados Unidos, Coreia, Índia e Brasil, para dados coletados até o ano de 2016.

Figura 1 - Número de Publicações de Patente x Ano coletadas por meio da ferramenta VP, relacionadas à Produção de Biofertilizantes

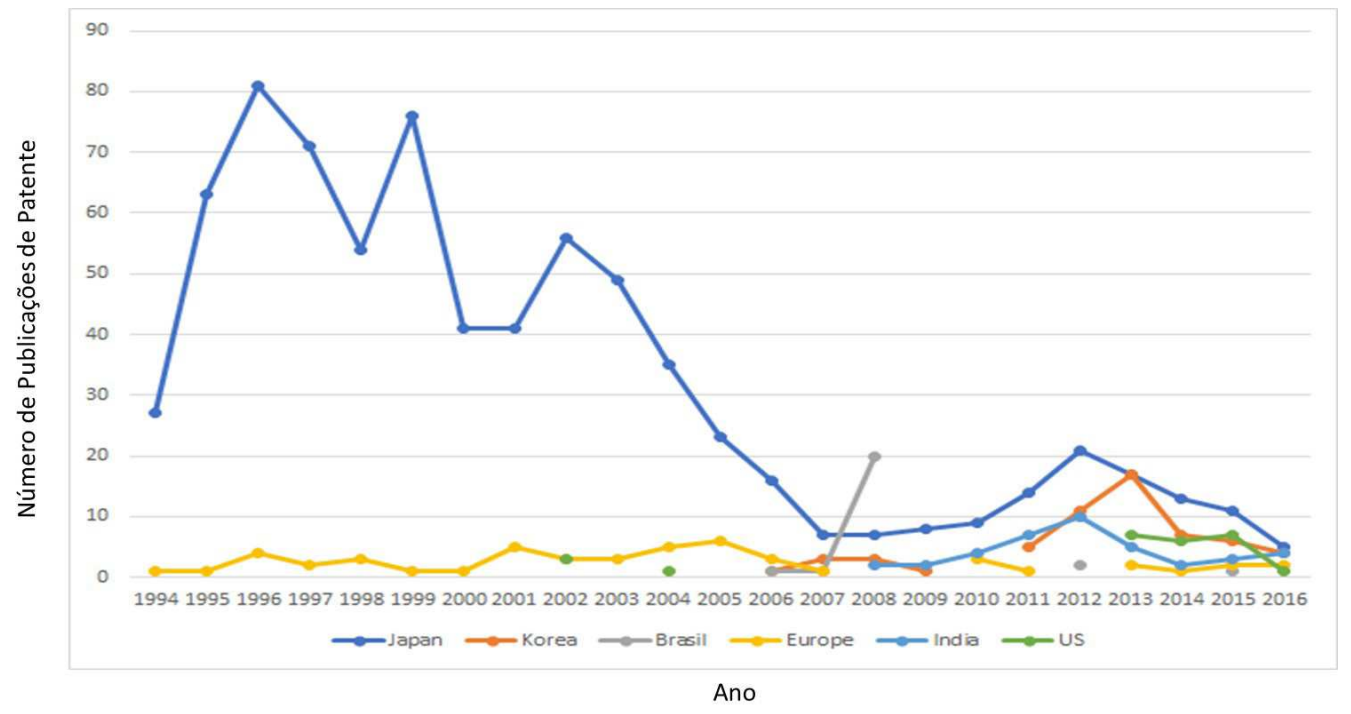

Fonte: Elaborada pelos autores deste artigo (2017, com dados gerados pelo VP 
Dentre os países relacionados na Figura 1, constata-se uma forte presença do Japão, quase que praticamente exclusiva, quanto à publicação de patentes associadas à utilização de resíduos para a produção de biofertilizantes entre os anos de 1994 a 2007.

O mercado de biofertilizantes asiático é o terceiro maior mercado mundial, e espera-se que atinja o segundo maior crescimento até o ano de 2020, com uma taxa de crescimento de $11,40 \%$ (BUSINESS WIRE, 2018).

Por meio de pesquisa de busca patentária realizada por meio do VP para o termo biofertilizer, verifica-se que o Japão apresenta 57 (cinquenta e sete) titulares de patentes, responsáveis por um total de 1.387 (mil trezentos e oitenta e sete) publicações de patentes. Do total de titulares de patentes, 95,53\% respondem por Empresas Privadas e 3,17\% por Institutos de Pesquisa.

Os 3 (três) principais depositantes japoneses são:

a) Hitachi Ltd: com 200 (duzentas) publicações de patente, sendo 38\% delas correspondentes ao código C05F e 28\% correspondentes ao código B09B;

b)Sanyo Electric Co Ltd: com 99 (noventa e nove) publicações de patente, sendo 33\% delas correspondentes ao código C05F e 33\% correspondentes ao código B09B;

c) Mitsubishi Electric Corp: com 92 (noventa e duas) publicações de patente, sendo 33\% delas correspondentes ao código C05F e 27\% correspondentes ao código B09B.

A Hitachi, Ltd. (Kabushiki Kaisha Hitachi Seisakusho) é um conglomerado multinacional, tendo sido fundada em 1910, e está presente na América do Sul desde o ano de 1940.

Sua sede localiza-se na cidade de Tóquio, no Japão. Atua em diversos segmentos, tendo como pilar a Inovação Social: tecnologia da informação; soluções em segurança; materiais industriais; equipamentos de radiodifusão; tecnologia de transmissão; infraestrutura; meio ambiente, energia, óleo e gás; maquinário e construção; automotivo e transporte; soluções em ar condicionado; medicina e saúde; biotecnologia e produtos automotivos (HITACHI, 2018).

A Inovação Social tem como premissa a busca por um amanhã sustentável por toda a humanidade, de forma global e colaborativa, com um novo olhar para a resolução de problemas sociais.

No âmbito da agricultura, por exemplo, a Hitachi Ltd. aborda a melhoria da produtividade no gerenciamento agrícola por meio da tecnologia GIS como forma de Inovação Social.

A Figura 2 apresenta a evolução temporal da principal depositante de patentes japonesa, a Hitachi Ltd, a qual responde por 200 (duzentos) publicações de patentes de um total de 1.387 (mil trezentos e oitenta e sete) patentes do país, correspondentes assim a $14,42 \%$ do total de publicações do país. 
Figura 2 - Número de Publicações de Patente x Ano coletadas por meio da ferramenta VP, relacionadas à Produção de Biofertilizantes - Hitachi Ltd

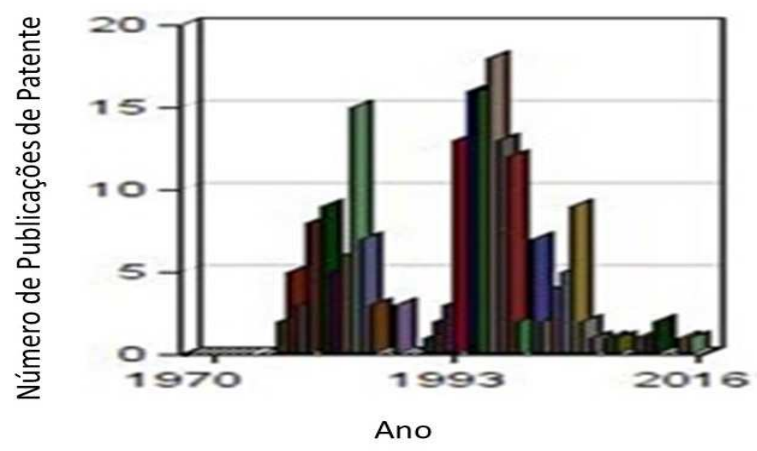

Fonte: Elaborada pelos autores deste artigo (2017), com dados gerados pelo VP

Através da Figura 2 verifica-se a forte atuação da Hitachi Ltd no segmento entre os anos de 1975 e 2016. A partir do ano de 2002 verifica-se que o Japão ainda se mostra predominante nesta área de atuação, porém com grande queda até o ano de 2007, e a partir daí apresenta-se com um número de publicações próximo ao de outros países, como a Coreia. Ainda, verifica-se que a Europa está sempre presente, apesar do baixo número evidenciado, com publicações de patentes desde o ano de 1994 até 2007.

O mercado europeu de biofertilizantes é o segundo maior mercado global, com uma expectativa de taxa de crescimento de 14,90\% até 2020 (BUSINESS WIRE, 2018).

Dentre os principais países responsáveis pela publicação de patentes no continente europeu, verificou-se que a Alemanha ocupa o primeiro lugar, com 139 publicações de patentes (33,33\% das publicações europeias), seguida por República Tcheca (67 publicações, 16,07\% das publicações europeias), Suíça (36 publicações, 8,63\%), Ucrânia, França, Finlândia, Suécia, Áustria, Reino Unido, Moldávia, Holanda, Dinamarca e Itália.

Por meio de pesquisa de busca patentária para o termo biofertilizer realizada por meio do VP, verificou-se que a Europa apresenta 51 (cinquenta e um) titulares de patentes responsáveis por um total de 417 (quatrocentos e dezessete) publicações de patentes, sendo os 4 (quatro) principais depositantes:

a) Herhof Umwelttechnik GmbH: com 29 (vinte e nove) publicações de patente, sendo $42 \%$ delas correspondentes ao código C05F e 11\% correspondentes ao código B09B;

b) BASF AG: com 25 (vinte e cinco) publicações de patente;

c) Ceska Zemedelska Univerzita V Praze: com 15 (quinze) publicações de patente, sendo $53 \%$ delas correspondentes ao código C05F e 12\% correspondentes ao código B09B;

d)Institute of Agricultural Microbiology and Agro-Industrial Manufacture of the Ukrainian Academy of Agrarian Sciences: com 15 (quinze) publicações de patente, sendo $21 \%$ delas correspondentes ao código $\mathrm{C} 05 \mathrm{~F}$.

A principal empresa responsável por publicações de patentes na Europa, a Herhof Umwelttechnik $\mathrm{GmbH}$, é uma empresa alemã, mais especificamente uma sociedade com responsabilidade limitada (tradução do alemão do termo $\mathrm{GmbH}$ ). Não se encontra mais em operação, tendo sido substituída pela Herhof $\mathrm{GmbH}$. 
Atua no segmento de projeto, construção e operação de plantas de compostagem, com mais de 40 (quarenta) plantas distribuídas em países como a Alemanha, Áustria, Itália, Espanha, Bélgica, Polônia, Canadá e Escócia. Os sistemas disponibilizados pela empresa transformam resíduos orgânicos em subprodutos em um período de 7 (sete) a 10 (dez) dias, por meio do seu aquecimento a $65^{\circ} \mathrm{C}$ (HERHOF GMBH, 2018).

A Figura 3 apresenta a evolução temporal da principal depositante de patentes europeia, a Herhof Umwelttechnik Gmbh, a qual responde por 29 (vinte e nove) publicações de patentes de um total de 417 (quatrocentos e sete) patentes do país, correspondentes a 6,95\%.

Figura 3 - Número de Publicações de Patente x Ano coletadas por meio da ferramenta VP, relacionadas à Produção de Biofertilizantes - Herhof Umwelttechnik Gmbh

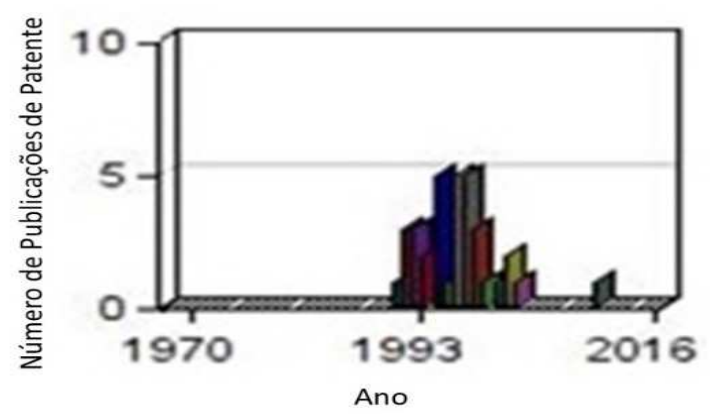

Fonte: Elaborada pelos autores deste artigo (2017), com dados gerados pelo VP

Por meio da análise dos resultados da Figura 3, é possível verificar um maior número de publicações de patentes entre o final da década de 80 e início do século XXI.

A BASF AG é uma sociedade por ações alemã (tradução para Aktiengesellschaft), segunda principal empresa europeia responsável por publicações de patentes. Foi convertida posteriormente para uma Companhia Europeia, a BASF SE, resultante do olhar da instituição para o mercado global (BASF, 2007).

A partir de 2006, tanto o Brasil quanto a Coreia passam a publicar patentes na área de utilização de resíduos para a produção de fertilizantes. Contudo, é interessante verificar que, enquanto outros países apresentaram um decréscimo de publicações nesse período, o Brasil, em oposição, supera o Japão e os outros países entre os anos de 2007 e 2008.

Por meio de pesquisa de busca patentária realizada por meio do VP para o termo biofertilizer, verifica-se que o Brasil apresenta 03 (três) titulares de patentes, responsáveis por um total de 40 (quarenta) patentes depositadas. Do total de titulares de patentes, 82,50\% respondem por Empresas Privadas e 17,50\% por Fundações.

Os 3 (três) depositantes do Brasil são:

a) Sergio Massao Watanabe: com 25 (vinte e cinco) publicações de patente, sendo $14 \%$ delas correspondentes ao código $\mathrm{C} 05 \mathrm{~F}$;

b) Capixaba Couros Ltda: com 08 (oito) publicações de patente, sendo $13 \%$ delas correspondentes ao código C05F;

c) Fundação de Amparo à Pesquisa e Inovação do Espírito Santo (FAPES): com 07 (sete) publicações de patente, sendo 14\% delas correspondentes ao código C05F. 
A totalidade das 40 (quarenta) publicações de patente brasileiras depositadas levantadas pelo software VP referem-se à classificação de fabricação de produtos químicos básicos, fertilizantes e compostos de nitrogênio, plásticos e borracha sintética em formas primárias.

Verifica-se que 33 (trinta e três) das publicações de patente estão relacionadas a Empresas Privadas, enquanto 07 (sete) delas a uma Fundação.

A Figura 4 apresenta a evolução temporal da principal depositante de patentes do Brasil, Sergio Massao Watanabe, a qual responde por 25 (vinte e cinco) publicações de patente de um total de 40 (quarenta) publicações de patente do país, correspondentes a $62,50 \%$, entre meados de 2006 e 2008.

Figura 4 - Número de Publicações de Patente x Ano coletadas por meio da ferramenta VP, relacionadas à Produção de Biofertilizantes - Sergio Massao Watanabe

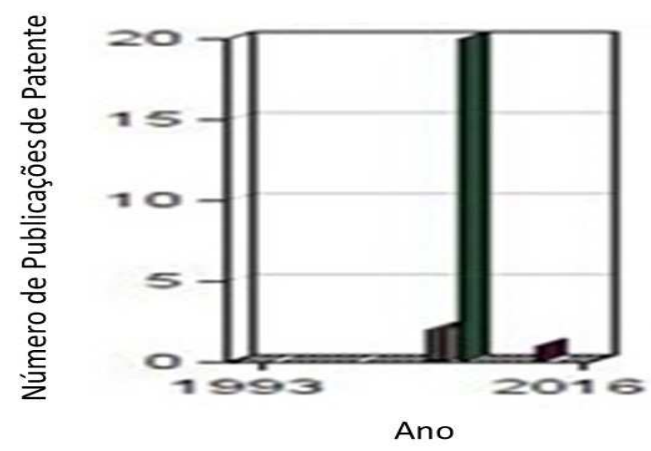

Fonte: Elaborada pelos autores deste artigo (2017), com dados gerados pelo VP

Em um caso de sucesso relatado pelo SEBRAE (2017), é possível verificar o benefício de uma das patentes inventadas por Sérgio Massao Watanabe, a biotecnologia denominada HSNI, a qual transforma matéria orgânica em adubo em um período de apenas 15 (quinze) dias, quando convencionalmente o período é de 100 (cem) dias.

O lixo coletado é misturado ao "bio-extrato" de nome científico "HSNI", conjunto de bactérias (fungos, leveduras e outros organismos vivos) que aceleram o processo de compostagem realizado a céu aberto, sem a geração de chorume ou emissão de gases de efeito estufa. A ação dura 15 dias, enquanto por métodos convencionais o prazo varia entre dois e três meses. A partir daí, tem-se o adubo a ser comercializado $(\mathrm{O}$ PROGRESSO, 2013, p. 2).

A biotecnologia é patenteada por Sérgio Massao Watanabe, diretor do Grupo Organoeste, que a fornece para outras empresas licenciadas, de mesmo nome, em regiões do país. Começou a ser desenvolvida no Japão e foi finalizada no Brasil, com a ajuda da USP - Universidade de São Paulo.

A empresa Organoeste Campo Grande, situada na cidade de mesmo nome, no Mato Grosso do Sul, atua desde 2005 no ramo de Logística Reversa, com a transformação do material orgânico recebido das indústrias em adubo, com uma clientela de 30 (trinta) geradores (restaurantes, fábricas, propriedades rurais e frigoríficos) que pagam pelo descarte de seus rejeitos. Já o adubo orgânico produzido dos rejeitos é comprado por aproximadamente 800 (oitocentos) produtores rurais (SEBRAE, 2017). Vale ressaltar, contudo, que todo rejeito, antes de ser aproveitado 
pelo processo de transformação em adubo orgânico, deve ter amostras submetidas a exames laboratoriais para verificar a compatibilidade com relação à biotecnologia HSNI (ASN, 2012).

$\mathrm{O}$ custo da tonelada de adubo químico pode chegar a $\mathrm{R} \$ 1.200,00$, enquanto o adubo orgânico varia de $\mathrm{R} \$ 120,00$ (tonelada a granel) a $\mathrm{R} \$ 180,00$ (tonelada ensacada) (SEBRAE, 2017).

A Figura 5 apresenta a evolução temporal da segunda principal depositante de patentes do Brasil, Capixaba Couros Ltda., a qual responde por 8 (oito) publicações de patente de um total de 40 (quarenta) publicações de patente do país, correspondentes a 20\%. Na Figura verifica-se que as patentes foram publicadas recentemente.

Figura 5 - Número de Publicações de Patente x Ano coletadas por meio da ferramenta VP, relacionadas à Produção de Biofertilizantes - Capixaba Couros Ltda.

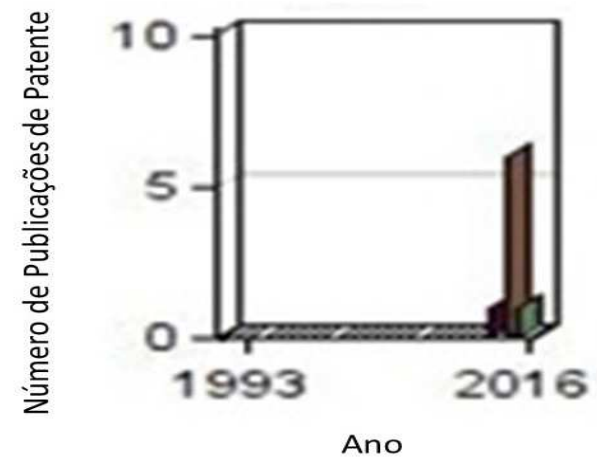

Fonte: Elaborada pelos autores deste artigo (2017), com dados gerados pelo VP

A Capixaba Couros Ltda., empresa localizada em Baixo Guandu, no Espírito Santo, é citada em dissertação de Mestrado, na qual o lodo de curtume desidratado, cedido pela empresa, é aplicado em testemunhas amostrais com diferentes percentuais de mistura para a produção de mudas do limoeiro "Cravo" (TUDEIA, 2016). Durante a pesquisa foram verificados outros trabalhos em que a empresa contribui com a disponibilidade do referido lodo para estudos técnicos, demonstrando a relação entre esta Microempresa e Instituições de Ensino Superior.

Importante registrar a parceria entre a Fundação de Amparo à Pesquisa e Inovação do Espírito Santo (FAPES), Instituto Federal do Espírito Santos (IFES) e a empresa Capixaba Couros Ltda. em processos de reaproveitamento de resíduo de curtume industrial para a agricultura, em culturas como café, milho, pastagens, fruteira e eucalipto. Tal parceria objetiva tanto promover o desenvolvimento tecnológico, evidenciado por artigos científicos, patentes e aplicações práticas, quanto subsidiar a criação de normativas de utilização de resíduos, sendo uma clara experiência relacionando uma Fundação, uma Instituição de Nível Superior e uma Empresa direcionada à resolução de problemas gerados pelo resíduo de curtume (FAPES, 2015).

A Figura 6 apresenta a evolução temporal da terceira principal depositante de patentes do Brasil, a Fundação de Amparo à Pesquisa e Inovação do Espírito Santo (FAPES), a qual responde por 08 (oito) publicações de patente de um total de 40 (quarenta) publicações de patente do país, correspondentes a $20 \%$. Na Figura verifica-se que as patentes foram publicadas recentemente. 
Figura 6 - Número de Publicações de Patente x Ano coletadas por meio da ferramenta VP, relacionadas à Produção de Biofertilizantes - Fundação de Amparo à Pesquisa e Inovação do Espírito Santo

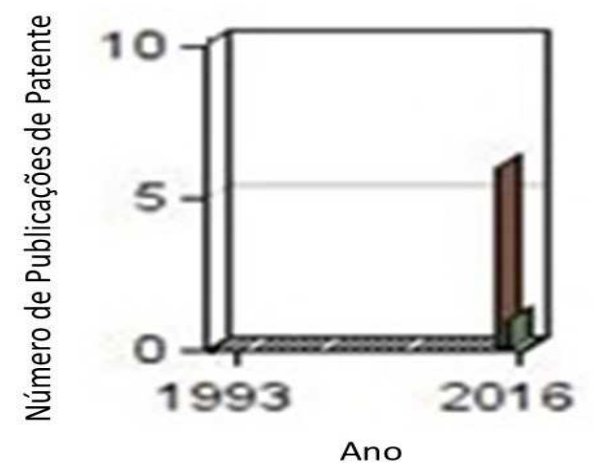

Fonte: Elaborada pelos autores deste artigo (2017), com dados gerados pelo VP

A Coreia apresenta um bom ritmo de publicação de patentes a partir de 2011 e, no ano de 2013, atingiu um nível de publicações equivalente ao Japão, com queda a partir daí.

Por meio de pesquisa de busca patentária realizada por meio do VP para o termo biofertilizer, verifica-se que a Coreia apresenta 4 (quatro) titulares de patentes responsáveis por um total de 52 (cinquenta e duas) publicações de patente do país. Neste país verifica-se um comportamento distinto em relação aos demais países analisados: do total de titulares de patentes, 42,31\% respondem pelo Governo, 38,46\% por Empresas Privadas e 19,23\% por Fundações.

Os 4 (quatro) depositantes da Coreia são apresentados a seguir:

a) Republic of Korea (Management: Rural Development Administration): com 22 (vinte e duas) publicações de patente, sendo $28 \%$ delas correspondentes ao código C05F e 3\% correspondentes ao código B09B;

b) Eco Bio Co.: com 10 (dez) publicações de patente, sendo $45 \%$ delas correspondentes ao código C05F e 5\% correspondentes ao código B09B;

c) Hoei Bussan KK: com 10 (dez) publicações de patente, sendo 31\% delas correspondentes ao código C05F e 26\% correspondentes ao código B09B;

d) Knu-Industry Cooperation Foundation: com 10 (dez) publicações de patente, sendo 9\% delas correspondentes ao código C05F.

Por meio da sua análise verifica-se a totalidade de 52 (cinquenta e duas) publicações de patentes $(100 \%)$ associadas à classificação Fabricação de produtos químicos básicos, fertilizantes e compostos de nitrogênio, plásticos e borracha sintética em formas primárias.

Vale ressaltar que além da forte presença do segmento de fertilizantes, registra-se a presença de classificações associadas aos segmentos de pesticidas e agroquímicos, médico e odontológico, farmacêutico, alimentício e de fabricação de máquinas. Entretanto, aquelas que realmente importam para os resultados da pesquisa, referentes aos biofertilizantes, referem-se à classificação Fabricação de produtos químicos básicos, fertilizantes e compostos de nitrogênio, plásticos e borracha sintética em formas primárias.

A Figura 7 apresenta a evolução temporal da principal depositante de patentes da Coreia, Republic of Korea (Management: Rural Development Administration). 
Figura 7 - Número de Publicações de Patente x Ano coletadas por meio da ferramenta VP, relacionadas à Produção de Biofertilizantes - Republic of Korea (Management: Rural Development Administration)

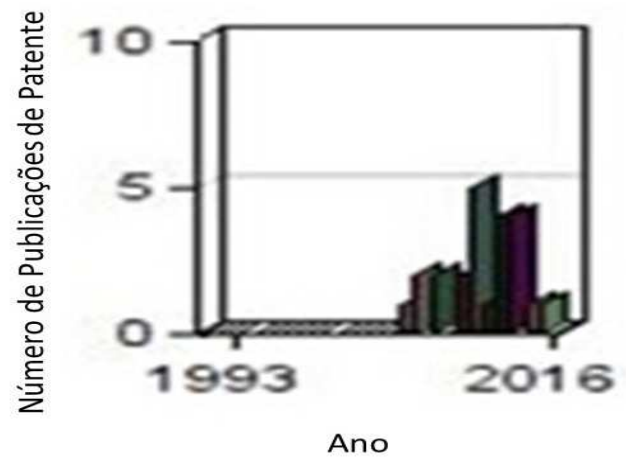

Fonte: Elaborada pelos autores deste artigo (2017), com dados gerados pelo VP

A Republic of Korea (Management: Rural Development Administration) responde por 22 (vinte e duas) publicações de patente de um total de 52 (cinquenta e duas) publicações de patente do país, correspondentes a 42,31\%. Verifica-se por meio da Figura uma elevada concentração de publicações recentes para a instituição.

A partir de 2008, a Índia surge no cenário, com publicações de patentes entre o ano de 2008 e 2016, com um pico de publicações no ano de 2012. Os Estados Unidos destacam-se entre os anos de 2013 e 2016.

O mercado norte-americano de biofertilizantes é o maior mercado global, com uma taxa de crescimento de 16,65\% até 2020 (BUSINESS WIRE, 2018).

A Figura 8 apresenta a variação proporcional do consumo em toneladas de biofertilizantes dos Estados Unidos, por tipo de produto: fixadores de nitrogênio, solubilizadores de fosfato, mobilizador de potássio e outros tipos entre 2016 e 2024.

Figura 8 - Proporção do mercado de biofertilizantes dos EUA, por produto - 2016 e 2024 (toneladas)

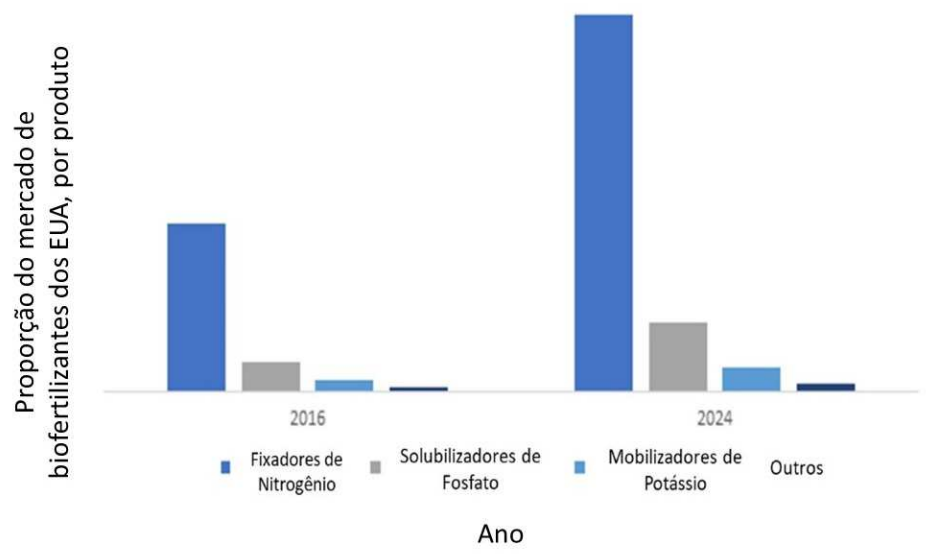

Fonte: GLOBAL MARKET INSIGHTS (2018)

Proporcionalmente, o mercado de fixadores de nitrogênio apresenta-se muito maior que para os outros componentes em razão da persistência do fertilizante à base de nitrogênio no solo ser menor que os fertilizantes à base de fósforo e potássio, o que faz com que a necessidade de produção deste insumo seja maior. 
Por meio de pesquisa de busca patentária realizada por meio do VP para o termo biofertilizer, verifica-se que os Estados Unidos apresentam 8 (oito) titulares de patentes responsáveis por um total de 56 (cinquenta e seis) publicações de patente. Do total de titulares de patentes, $50,00 \%$ respondem por Empresas Privadas, 26,79\% pelo Governo e 23,21\% por Universidades.

Os 3 (três) principais depositantes dos Estados Unidos são:

a) The Procter \& Gamble Company: com 11 (onze) publicações de patente, sendo $41 \%$ delas correspondentes ao código C05F e 23\% correspondentes ao código B09B;

b) The United States of America, as represented by the Secretary of Agriculture: com 09 (nove) publicações de patente, sendo $36 \%$ delas correspondentes ao código C05F;

c) Cornell University: com 07 (sete) publicações de patente, sendo 36\% delas correspondentes ao código $\mathrm{C} 05 \mathrm{~F}$.

Por meio da sua análise verifica-se a totalidade de 56 (cinquenta e seis) publicações de patentes $(100 \%)$ associadas à classificação Fabricação de produtos químicos básicos, fertilizantes e compostos de nitrogênio, plásticos e borracha sintética em formas primárias.

Vale ressaltar que além da forte presença do segmento de fertilizantes, registra-se a presença de classificações associadas aos segmentos de pesticidas e agroquímicos, médico e odontológico, farmacêutico, alimentício e de fabricação de máquinas. Entretanto, aquelas que realmente importam para os resultados da pesquisa, referentes aos biofertilizantes, referem-se à classificação Fabricação de produtos químicos básicos, fertilizantes e compostos de nitrogênio, plásticos e borracha sintética em formas primárias.

A Figura 9 apresenta a evolução temporal da principal depositante de patentes dos Estados Unidos, a The Procter \& Gamble Company.

Figura 9 - Número de Publicações de Patente x Ano coletadas por meio da ferramenta VP, relacionadas à Produção de Biofertilizantes - The Procter \& Gamble Company

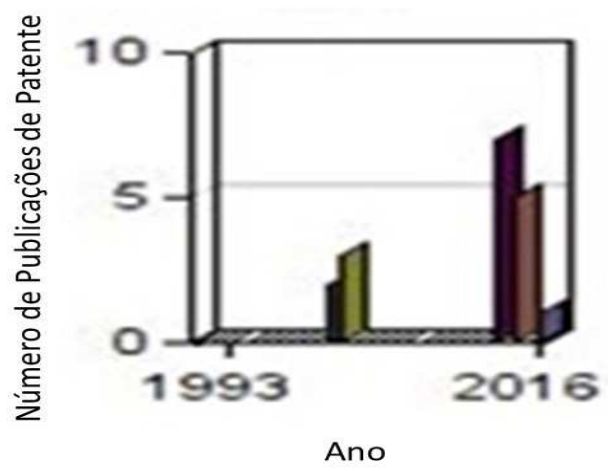

Fonte: Elaborada pelos autores deste artigo (2017), com dados gerados pelo VP

É possível verificar por meio da Figura 9 que a The Procter \& Gamble Company publicou patentes em meados do ano 2000, porém passou a atuar com mais ênfase na publicação nos últimos anos. Responde por 11 (onze) publicações de patente de um total de 56 (cinquenta e seis) patentes do país, correspondentes a $19,64 \%$. 


\section{Considerações Finais}

O Japão, Europa, Estados Unidos, Coreia, Índia e Brasil encontram-se entre os países com maior proeminência no âmbito de publicações de patentes para o manejo de resíduos destinado à produção de biofertilizantes. Destacam-se as seguintes instituições japonesas: Hitachi Ltd; Sanyo Electric Co Ltd; Mitsubishi Electric Corp. Do total de titulares de patentes japonesas, 95,53\% respondem por Empresas Privadas e 3,17\% por Institutos de Pesquisa.

Esta afirmação é ratificada diante de um total de 1.952 publicações de patentes (100\%) associadas à classificação Fabricação de produtos químicos básicos, fertilizantes e compostos de nitrogênio, plásticos e borracha sintética em formas primárias, seguida por classificações associadas à fabricação de máquinas.

Vale ressaltar que além da forte presença do segmento de fertilizantes, registra-se a presença de classificações associadas aos segmentos médico e odontológico, farmacêutico, alimentício, petrolífero, de transporte, da construção civil, de cosméticos e bebidas. Entretanto, aquelas que realmente importam para os resultados da pesquisa, referentes aos biofertilizantes, referem-se à classificação Fabricação de produtos químicos básicos, fertilizantes e compostos de nitrogênio, plásticos e borracha sintética em formas primárias.

Um detalhamento mais apurado das patentes ora verificadas tornará possível a identificação de tecnologias relevantes, inovações incrementais, nichos de mercado para atuação e nível maturidade tecnológica, especialmente se comparado ao número de artigos publicados por país.

Não apenas é importante a análise das patentes estabelecidas, mas também a análise dos próprios players e de suas estratégias. Um exemplo a ser citado é o da empresa Hitachi, com a estratégia da Inovação Social por meio da Tecnologia da Informação e da Internet das Coisas aplicada ao Agronegócio.

Através dos dados de séries históricas contidos no presente documento espera-se, assim, subsidiar estratégias de atuação pautadas nas ações de inovação, tecnologia e sustentabilidade demandadas pela sociedade e municiar os tomadores de decisão com informações que permitam promover políticas e legislações destinadas ao estímulo do empreendedorismo nacional $e$ à geração de oportunidades de negócio.

\section{Referências}

ASN - Agência Sebrae de Notícias. Biotecnologia exige análise laboratorial dos resíduos, antes de transformá-los em adubo orgânico. ASN: Minas Gerais, 2012. Disponível em: < http:// www.mg.agenciasebrae.com.br/sites/asn/uf/MG/biotecnologia-exige-analise-laboratorial-dos-residuosantes-de-transforma-los-em-adubo-organico,0ceb0101df816410VgnVCM1000003b74010aRCRD>. Acesso em: 03 dez. 2017.

BARMAN, Manashi; PAUL, Srijita; CHOUDHURY, Aditi Guha; ROY, Pinaki; SEN, Jahnavi. Biofertilizer as Prospective Input for Sustainable Agriculture in India. International Journal of Current Microbiology and Applied Sciences, v. 6, n. 11, p. 1.177-1.186, 2017. Disponível em: <https://www.ijcmas.com/6-11-2017/Manashi\%20Barman,\%20et\%20al.pdf>. Acesso em: 13 jan. 2018. 
Europaea, SE) with the company name BASF SE. 2007, 141 p. Disponivel em: <https:// www.basf.com/documents/corp/en/about-us/publications/reports/2007/Conversion_BASF_AG_to_ SE.pdf > . Acesso em: 12 jan. 2018.

BUSINESS WIRE. Global Biofertilizer Market 2016-2020 - Main Growth Driver is Affordable Cost of Bio Fertilizers - Research and Markets. Disponível em: <https:/www.businesswire.com/ news/home/20160426005882/en/Global-Biofertilizer-Market-2016-2020---Main-Growth > . Acesso em: 13 jan. 2018.

DA COSTA, Leticia Magalhães; E SILVA, Martim Francisco de Oliveira. A indústria química e o setor de fertilizantes. BNDES 60 Anos - Perspectivas Setoriais. Rio de Janeiro: BNDES, 2012. Disponível em: <https://www.bndes.gov.br/SiteBNDES/export/sites/default/bndes_pt/Galerias/ Arquivos/conhecimento/livro60anos_perspectivas_setoriais/Setorial60anos_VOL2Quimica.pdf > . Acesso em: 19 nov. 2017.

FAPES. Fapes apoia projeto que busca o reaproveitamento em resíduo industrial. 2015. Disponível: <https://fapes.es.gov.br/fapes-apoia-projeto-que-busca-o-reaproveitame > . Acesso em: 15 jan. 2017.

GLOBAL MARKET INSIGHTS. Biofertilizers market size to reach $\$ 1.66$ billion by 2022 : Global Market Insights, Inc. Disponível em: <https://www.gminsights.com/>. Acesso em: 13 jan. 2018.

HERHOF GMBH. Composting System. Disponível em: < http://www.herhof.com/en/products/ composting-system.html >. Acesso em: 13 jan. 2018.

HITACHI. HITACHI - Inspire the next. Disponível em: <http://www.hitachi.com/>. Acesso em: 14 jan. 2018.

IAEA. Biofertilizer Technology in Pakistan. Lab to Field: A success story of Biofertilizer Technology for crop nutrients in Pakistan. Disponível em: <www-naweb.iaea.org/nafa/ ews/ Biofertilizer-brochure.pptx>. Acesso em: 13 jan. 2018.

INPI. Inventando o futuro: uma introdução às patentes para as pequenas e médias empresas. Rio de Janeiro: INPI, 2013.

Classificação de Patentes. Disponível em: <http://www.inpi.gov.br/menu-servicos/ patente/classificacao-de-patentes >. Acesso em: 13 jan. 2018.

NETTO, A. G.; DIAS, J. M. C. S. Política energética para a agricultura. In: Simpósio sobre energia na agricultura, tecnologias poupadoras de insumos, integração de sistemas energéticos e produção de alimentos, 1., 1984, Jaboticabal. FCAV/UNESP, 1984. p. 3-22.

NGUMAH, Chima; OGBULIE, Jude; ORJI, Justina; AMADI, Ekpewerechi. Potential of Organic Waste for Biogas and Biofertilizer Production in Nigeria. Environmental Research, Engineering and Management, n. 1(63), p.. 60-66, 2013. Disponível em: <http://erem.ktu.lt/index.php/erem/ article/viewFile/2912/2415 > . Acesso em: 13 jan. 2018.

O PROGRESSO. Empretec faz empresário investir em negócio sustentável no Estado. O Progresso: Dourados, 2013. 3 p. Disponível em: <http://www.progresso.com.br/caderno-a/meioambiente/empretec-faz-empresario-investir-em-negocio-sustentavel >. Acesso em: 04 dez. 2017.

SEBRAE. Casos de Sucesso: Organoeste Campo Grande. Disponível em: < http://sustentabilidade. sebrae.com.br/sites/Sustentabilidade/Para\%E2\%80\%93sua\%E2\%80\%93Empresa/Casos\%E2\%80\%9 3de\%E2\%80\%93sucesso/Organoeste-Campo-Grande>. Acesso em: 02 dez. 2017. 
TUDEIA, Tacisio Nunes. Lodo de curtume como alternativa na produção de mudas do portoenxerto limoeiro “Cravo”. 2016. 66 p. Disponível em: < http://uenf.br/posgraduacao/producaovegetal/wp-content/uploads/sites/10/2016/06/DISSERTA\%C3\%87\%C3\%83O.pdf>. Acesso em: 15 jan. 2018.

WIPO. C - Chemistry; Metallurgy. Disponível em: <http:/www.wipo.int/classifications/ipc//en/ ITsupport/Version20170101/transformations/ipc/20170101/en/htm/C05F.htm>. Acesso em: 15 abr. 2017.

\section{Sobre os autores}

\section{Eduardo Cardoso Garrido}

E-mail: eduardocardosogarrido@gmail.com

Graduado em Engenharia Sanitária e Engenharia Ambiental pela Escola Politécnica da UFBA. Graduado em Engenharia de Segurança do Trabalho pela Universidade Estácio de Sá. Especialista em Gestão Ambiental com Tecnologias Limpas pela Faculdade Área 1/ DeVry Brasil. Graduando em Direito pela Faculdade de Direito da UFBA. Mestre em Propriedade Intelectual e Transferência de Tecnologia para Inovação pela UFBA. Mestrando em Gestão da Inovação e Desenvolvimento Industrial pelo Centro Universitário SENAI CIMATEC.

Endereço Profissional: SEBRAE/BA. Rua Horácio César, 64, Dois de Julho. Salvador/BA. CEP: 40.060-350.

\section{Ângela Machado Rocha}

E-mail: anmach@gmail.com

Doutora em Energia e Ambiente pela Universidade Federal da Bahia (2013).

Endereço Profissional: Instituto de Saúde Coletiva da UFBA. Rua Basílio da Gama, 316 - Canela, Salvador/BA. CEP: 40.110-040.

\section{Douglas Alves Santos}

E-mail: dsaints@inpi.gov.br

Graduado em Engenharia Química (UFPE, 1999). Mestre em Engenharia de Processamento Químico de Petróleo e Gás Natural p ela mesma UFPE (2002). Doutor em Tecnologias de Processos Químicos e Bioquímicos da Escola de Química (EQ) da Universidade Federal do Rio de Janeiro (UFRJ, 2014).

Endereço Profissional: INPI. Rua Marechal Deodoro, 344, $8^{\circ}$ andar - Edifício Atalaia - Centro, Curitiba/PR - CEP: 80.010-010.

\section{Jose Miguel Vicente Gomila}

E-mail: vicente@triz.es

Specialties: Innovation, Conceptual Innovation, TRIZ, Technology Intelligence, Patents, R\&D Portfolio Management, R\&D Project Management,Techmining, GoldFir e Innovator, Ideal Matrix SW, TriSolver, Function Oriented Trends. Endereço Profissional: Universitat Politècnica de València. Camí de Vera, s/n, 46022 València, Espanha. 